\title{
Simulation du phénomène de talonnage en perçage vibratoire auto-entretenu
}

\author{
Nicolas GuiberT ${ }^{1, a}$, Henri Paris ${ }^{1}$ et Jö̈L ReCH ${ }^{2}$ \\ 1 Laboratoire G-SCOP, 46 avenue Félix Viallet, 38000 Grenoble, France \\ 2 Laboratoire LTDS, ENISE, 58 avenue Jean Parot, 42000 Saint-Étienne, France
}

Reçu le 15 mars 2007, accepté le 6 juillet 2007

\begin{abstract}
Résumé - L'augmentation de la productivité des opérations de perçage est un problème industriel qui existe depuis longtemps. Un des facteurs limitants dans les opérations de perçage est l'évacuation du copeau dès que le trou devient trop profond. La mauvaise évacuation du copeau entraîne souvent une mauvaise qualité de la surface usinée et le bris de l'outil. Pour résoudre ce problème, une technique de perçage vibratoire à basse fréquence auto-entretenu a été développée et dont le but est une meilleure fragmentation du copeau afin d'améliorer son évacuation. Pour cela, un porte-outil de perçage spécifique a été mis en place. La génération naturelle des vibrations de l'outil est fortement conditionnée par le choix des conditions de coupe et de la géométrie du foret. De plus, les phénomènes de talonnage perturbent le bon fonctionnement de la tête en amortissant les vibrations. Pour prédire le comportement du porte-outil de perçage vibratoire, une modélisation des différents efforts issus des différents phénomènes de coupe le long de l'arête du foret ainsi que la génération de la surface usinée a été effectuée. Ces modèles sont maintenant intégrés dans un simulateur numérique permettant d'étudier, en particulier, l'influence du talonnage sur le comportement du porte-outil de perçage vibratoire. Différentes campagnes d'essais sont réalisées pour permettre la validation des différents modèles intégrés dans le simulateur.
\end{abstract}

Mots clés : Perçage / vibrations auto-entretenues / modèles / talonnage / simulation

\begin{abstract}
Simulation of the ploughing effect on self vibratory drilling. The increase of productivity of the drilling operations is a major problem that exists for long time in production. The poor removal of chips in deep drilling of small diameter is often the cause of tool breakage and poor quality surface. The vibratory drilling enables the chip to be split thanks to the axial vibrations of the drill, self-maintained by the cutting energy. Thus chips are evacuated easily. A specific tool holder with an adapted axial stiffness has been developed. The cutting conditions are chosen to be inside the instable domain and with the chatter phenomenon the lip jumps out of the material. The parameters of the self-vibrating drilling head and the cutting conditions are conditioned by the presence of ploughing effect that dumps the vibrations. In order to foresee the behaviour of the drilling head, a numerical simulator that integrates the different forces models has been created. This simulator gives some information about the thrust force generated during the machining, the amplitude of the vibrations, the chip thickness and gives the possibility to study the influence of the cutting parameters on the vibrations. Some experiments have been made to validate the simulator.
\end{abstract}

Key words: Drilling / self-excited vibration / ploughing / chatter / numerical simulation

\section{Introduction}

Le monde de la fabrication a très souvent recours à des opérations de perçage et notamment des perçages de trous profonds (trous ayant un rapport L/D supérieur à 5) ou des opérations de forage. Le perçage est une opération

\footnotetext{
a Auteur pour correspondance :

nicolas.guibert@g-scop.inpg.fr
}

longue, donc coûteuse. Un des facteurs importants dans la durée de l'opération est le temps dédié à l'évacuation du copeau. Un copeau fractionné est une condition nécessaire pour que le perçage se déroule dans de bonnes conditions. En effet, un copeau long reste souvent bloqué au fond du trou entraînant une surpression locale (bourrage de copeau) et un échauffement important de l'outil qui peut mener à sa rupture. Des opérations de débourrage sont 


\section{Nomenclature}

\begin{tabular}{|c|c|}
\hline$A_{\mathrm{D}}$ & Aire du copeau $\left(\mathrm{mm}^{2}\right)$ \\
\hline$A_{\text {tal }}$ & Aire talonnée $\left(\mathrm{mm}^{2}\right)$ \\
\hline$c$ & Amortissement \\
\hline$d$ & Coefficient \\
\hline$F$ & Force de poussée $(\mathrm{N})$ \\
\hline$F_{\mathrm{z}}$ & Force axiale créée par la coupe dans la zone $1(\mathrm{~N})$. \\
\hline$F_{\mathrm{c}}^{1}, F_{\mathrm{c}}^{2}, F_{\mathrm{c}}^{3}$ & Effort de coupe dans les zones $1,2,3(\mathrm{~N})$ \\
\hline$F_{\text {tal }}$ & Force axiale créée par le talonnage $(\mathrm{N})$ \\
\hline$h$ & Épaisseur instantanée de copeau (mm) \\
\hline$k$ & Raideur (N.mm $\left.{ }^{-1}\right)$ \\
\hline$K_{1 \mathrm{t}}$ & Coefficient spécifique de coupe de la zone $1\left(\mathrm{~N} . \mathrm{mm}^{-2}\right)$ \\
\hline$K_{2 \mathrm{t}}$ & Coefficient spécifique de coupe de la zone $2\left(\mathrm{~N} . \mathrm{mm}^{-2}\right)$ \\
\hline$K_{3 \mathrm{t}}$ & Coefficient spécifique d'extrusion $\left(\mathrm{N} \cdot \mathrm{mm}^{-2}\right)$ \\
\hline$K_{\text {tal }}$ & Coefficient spécifique de talonnage (N.mm ${ }^{-3}$ ) \\
\hline$K_{\mathrm{v}}$ & Coefficient \\
\hline$l_{\mathrm{a}}$ & Longueur de l'arête de coupe (mm) \\
\hline$l_{1}$ & Largeur de la zone de coupe principale ( $\mathrm{mm})$ \\
\hline$l_{2}$ & Largeur de la zone de coupe secondaire (mm) \\
\hline$m$ & Masse mobile (kg) \\
\hline$N$ & Nombre de sous-divisions de l'arête de coupe \\
\hline$N_{\mathrm{c}}$ & Nombre d'arêtes de coupe \\
\hline$q$ & Coefficient pour le modèle de coupe \\
\hline$R$ & Rayon du foret (mm) \\
\hline$R_{2}$ & Rayon de la zone 2 (mm) \\
\hline$R_{3}$ & Rayon de la zone d'extrusion (mm) \\
\hline$V$ & Vitesse de coupe $\left(\mathrm{m} \cdot \mathrm{min}^{-1}\right)$ \\
\hline$V_{\text {zmax }}$ & Vitesse d'avance de l'outil $\left(\mathrm{m} \cdot \mathrm{min}^{-1}\right)$ \\
\hline$V_{\text {tal }}$ & Volume de talonnage $\left(\mathrm{mm}^{3}\right)$ \\
\hline$X_{0}, V_{0}, Z_{0}$ & Estimation de la position, vitesse et accélération de l'arête \\
\hline$\gamma$ & Angle de coupe du foret (degré) \\
\hline$\alpha$ & Angle de dépouille du foret (degré) \\
\hline$\delta$ & Angle de pointe du foret (degré) \\
\hline
\end{tabular}

donc la plupart du temps nécessaires pour assurer une bonne évacuation du copeau.

De nouvelles techniques permettant une meilleure évacuation des copeaux ont vu le jour ces dernières années. L'une d'elles est basée sur la fragmentation du copeau par vibrations axiales du foret. Dans [1], Gouskov a évalué l'amplitude et la fréquence des vibrations axiales d'un foret hélicoïdal compatibles avec une bonne fragmentation du copeau. Différents procédés permettant de créer ces vibrations existent actuellement. Suciu [2] présente un porte-outil spécifique dans lequel les vibrations axiales sont générées par la variation de l'épaisseur d'un film d'huile entre deux surfaces. Chabra [3] suggère de créer ces vibrations par l'intermédiaire des moteurs linéaires qui équipent les machines-outils modernes. Néanmoins toutes ces techniques nécessitent un apport extérieur d'énergie.

Une autre approche consiste à utiliser l'énergie de la coupe pour créer les vibrations axiales du foret via un porte-outil spécifique $[4,5]$. Cette technique permet ainsi de créer des vibrations sans aucun apport extérieur d'énergie. Si ces vibrations ont une amplitude plus importante que l'avance du foret, le copeau sera fractionné permettant ainsi une bonne évacuation par la goujure du foret. La difficulté est de maîtriser l'amplitude et la stabilité des vibrations. Le porte-outil spécifique (Fig. 1) est composé de 3 grandes parties : un système d'attachement de l'outil possédant une masse $m$ pouvant coulisser dans le corps principal qui réalise l'attachement entre le porte-outil et la machine. Enfin un ressort de raideur $k$ positionné entre le corps principal et la partie mobile du porte-outil permet d'accumuler l'énergie fournie par la coupe et de la restituer sous forme de vibrations axiales. Les vibrations sont la réponse directe du ressort à l'effort de poussée, l'effort de poussée étant entre autre fonction de la vitesse de coupe et de la vitesse d'avance $\mathrm{du}$ foret. La génération des vibrations est très fortement conditionnée par le choix de ces paramètres de coupe. Les vibrations sont de plus fortement perturbées par le phénomène de talonnage. L'utilisation de cette technique va permettre d'améliorer la productivité du fait de l'élimination de l'opération de débourrage et de l'utilisation de la lubrification. De nombreux secteurs industriels comme l'automobile ou l'aéronautique pourraient avoir recours à cette technologie. 


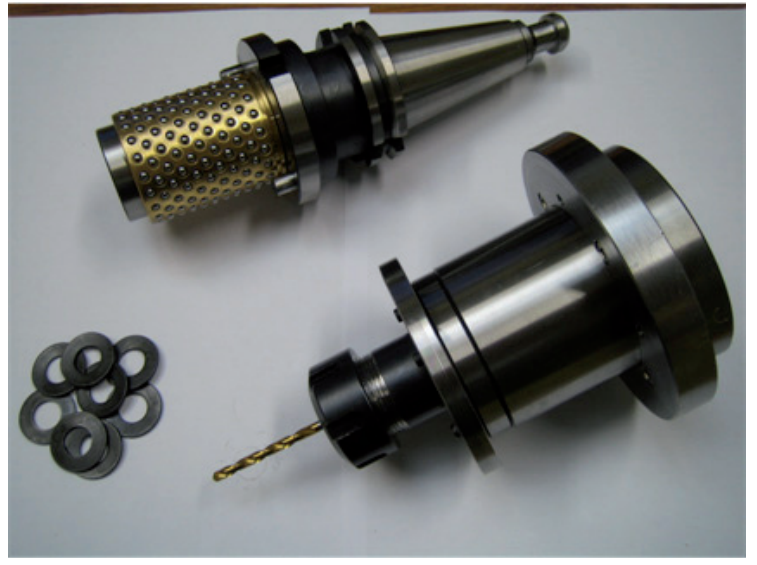

Fig. 1. Porte-outil spécifique de perçage vibratoire.

L'objectif de cet article est d'analyser par une approche numérique l'influence du talonnage sur les vibrations axiales du foret. Pour cela, il est nécessaire de modéliser l'ensemble du procédé (modèle dynamique du système usinant, modèle d'effort de coupe, modèle d'enlèvement de matière) puis de mettre en place un simulateur numérique intégrant ces différents modèles. Une campagne expérimentale permet de valider les résultats de simulation. Le simulateur servira pour l'étude de l'influence du talonnage sur les vibrations de l'outil et la forme du copeau.

\section{Modélisation}

La modélisation du perçage vibratoire nécessite :

- un modèle dynamique pour le comportement vibratoire du porte-outil;

- un modèle pour les efforts de poussée;

- un modèle d'enlèvement de matière.

\subsection{Le modèle dynamique}

Un ressort spécifique est positionné entre la partie fixe et la partie mobile de la tête de perçage. Ce ressort a une raideur maîtrisée et faible (entre 250 et $750 \mathrm{~N} . \mathrm{mm}^{-1}$ ). La raideur de ce ressort spécifique est donc beaucoup plus faible que la raideur radiale du foret. De plus, la raideur en torsion $\mathrm{du}$ foret est importante devant la raideur axiale du ressort monté dans le porte-outil de perçage vibratoire. Les vibrations générées par les phénomènes de torsion peuvent donc être négligées. Le comportement dynamique du porte-outil de perçage est alors modélisé [6] par un système de second ordre caractérisé par une masse $m$, une raideur $k$, et un amortissement $c$, excité par l'effort de poussée $F$ (Fig. 2). L'équation dynamique représentant le comportement de la tête s'écrit (Éq. (1)) :

$$
m . \ddot{x}+c . \dot{x}+k . x=F
$$

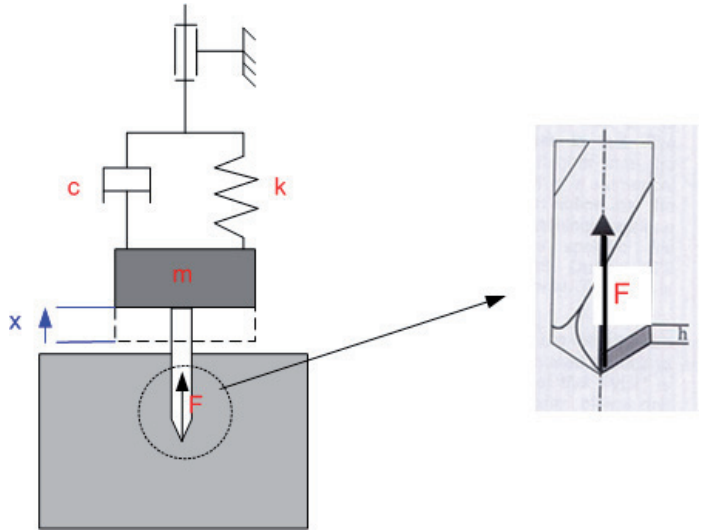

Fig. 2. Modélisation du porte-outil de perçage vibratoire.

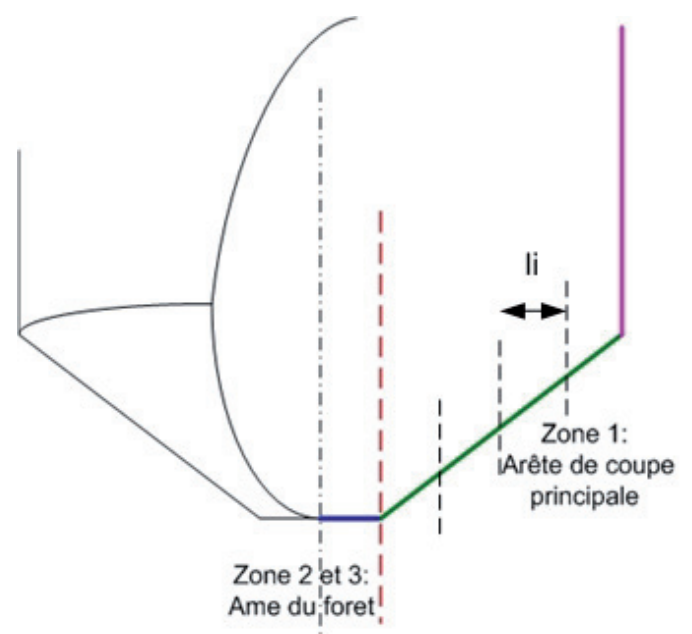

Fig. 3. Discrétisation d'un foret.

\subsection{Modèle d'effort de poussée}

Une détermination précise de l'effort de poussée $F$ est nécessaire pour modéliser finement le phénomène vibratoire. Le foret ne travaille pas de la même manière sur toute la longueur de l'arête de coupe. De ce fait, une discrétisation du foret est nécessaire.

\subsubsection{Discrétisation du foret en 3 zones}

L'arête de coupe d'un foret hélicoïdal peut être discrétisée en 3 parties (Fig. 3) où les phénomènes de coupe sont différents :

- la zone de coupe principale, «zone $1 »$, dans laquelle le phénomène de coupe est un phénomène d'enlèvement de matière;

- l'âme du foret qui peut se sous-décomposer en 2 parties (Fig. 4) :

- La zone de coupe secondaire, «zone 2 », où le phénomène de coupe est un phénomène d'enlèvement de matière mais avec des angles différents que ceux de la «zone $1 »$; 


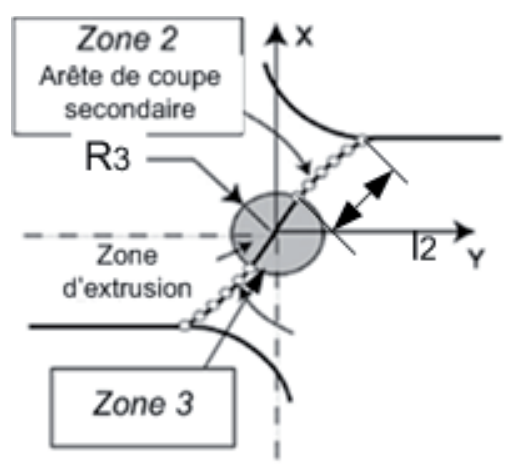

Fig. 4. Discrétisation de l'âme du foret.

- La zone centrale, «zone $3 »$. Cette partie du foret, de par sa géométrie et la faible vitesse de coupe, ne coupe pas la matière de la pièce mais la déforme vers les deux arêtes coupantes du foret. Il s'agit d'un phénomène d'extrusion.

Pour chacune de ces zones, un modèle de coupe peut être mis en place afin d'exprimer la force de poussée. En considérant une parfaite indépendance entre les différentes zones de l'outil, il est possible d'exprimer la force de poussée globale comme la somme des efforts créés dans chacune des zones (Éq. (2)).

$$
F=F_{\mathrm{c}}^{1}+F_{\mathrm{c}}^{2}+F_{\mathrm{c}}^{3}
$$

\subsubsection{Effort de poussée dans la zone de coupe principale}

Dans cette zone, l'effort de poussée résulte de deux phénomènes mécaniques : la force générée par l'enlèvement de matière $F_{\mathrm{z}}$ et la force générée par le talonnage $F_{\text {tal }}($ Éq. (3)).

$$
F_{\mathrm{c}}^{1}=F_{\mathrm{z}}+F_{\mathrm{tal}}
$$

\subsubsection{Modèle d'effort de coupe}

C'est dans cette «zone $1 »$ qu'a lieu la majorité de l'enlèvement de matière. En effet, l'arête de coupe pénètre dans le matériau entraînant le cisaillement de la matière et la formation d'un copeau. Ce phénomène génère un effort $F_{\mathrm{z}}$ fonction de la section du copeau $A_{\mathrm{D}}$ (Fig. 5). Dans le cas présent, la section du copeau peut s'exprimer en fonction de l'épaisseur instantanée du copeau $h$ et de la largeur de l'arête de coupe $l_{1}$. Yang [7] modélise cette force (Éq. (4)) pour laquelle $K_{1 \mathrm{t}}$ est la pression spécifique de coupe et $q$ un coefficient.

$$
F_{\mathrm{z}}=K_{1 \mathrm{t}} \cdot\left(h \cdot l_{1}\right)^{q}
$$

Stephenson et Bandyopadhyay [8] expriment le coefficient spécifique de coupe $K_{1 t}$ en fonction de la vitesse de coupe $V$ et de la géométrie de l'outil par l'intermédiaire de l'angle de coupe $\gamma$ (Éq. (5)).

$$
K_{1 \mathrm{t}}=K_{v} \cdot h^{a} \cdot V^{b} \cdot(1-\sin (\gamma))^{d}
$$

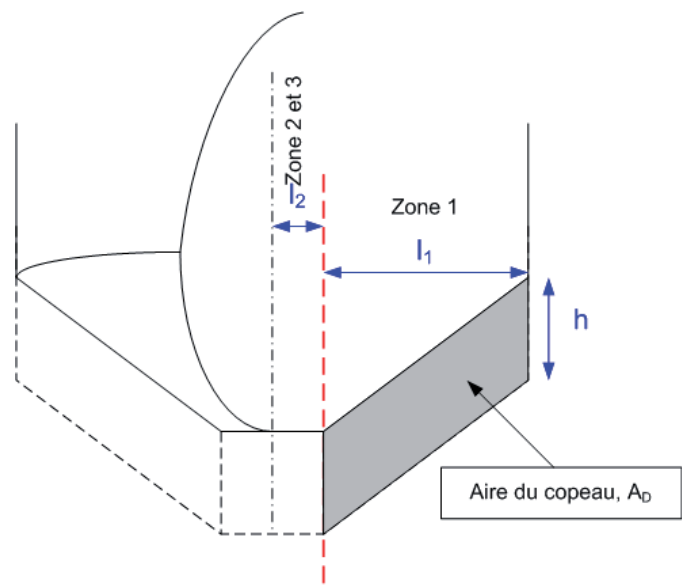

Fig. 5. Représentation de l'aire du copeau.

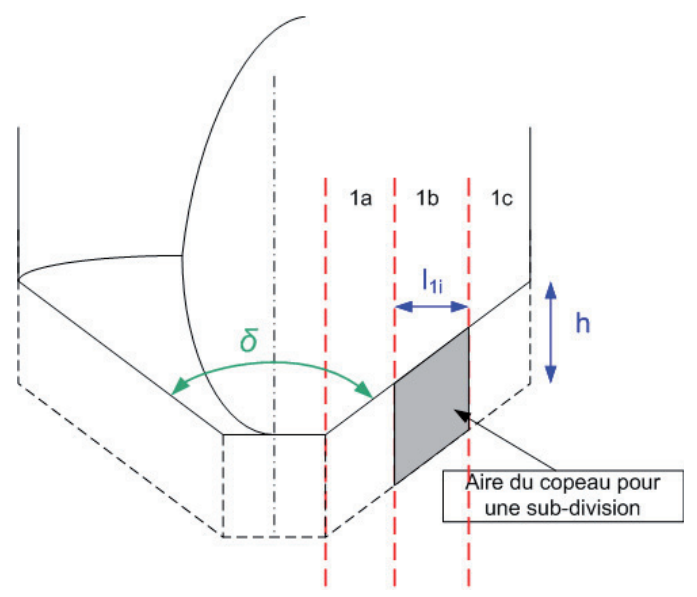

Fig. 6. Découpage de la zone de coupe principale.

Dans la zone de coupe principale, la vitesse de coupe est fonction du rayon et n'est donc pas constante. De plus, la géométrie de l'outil varie avec le rayon de l'outil (l'angle de coupe varie le long de l'arête). Il n'est donc pas possible de prendre un unique coefficient spécifique de coupe pour modéliser la coupe dans cette zone. Un découpage de cette zone en plusieurs segments est nécessaire (Fig. 6). Dans chacun de ces segments de largeur $l_{1 i}$, la vitesse de coupe et la géométrie d'outil sont supposées constantes, permettant de prendre un coefficient spécifique constant. L'effort de poussée généré dans chaque segment $i$ est calculé à l'aide de l'équation (6).

$$
\Delta F_{\mathrm{zi}}=K_{1 \mathrm{ti}} \cdot\left(h \cdot l_{1 \mathrm{i}}\right)^{q}
$$

En ne considérant aucune interaction entre les différentes zones, l'effort de poussée global dans la zone de coupe principale s'exprime comme la somme des efforts de poussée de chaque segment $i$ (Éq. (7)).

$$
F_{\mathrm{z}}=N_{\mathrm{c}} \sum_{i=1}^{N} \Delta F_{\mathrm{zi}}
$$




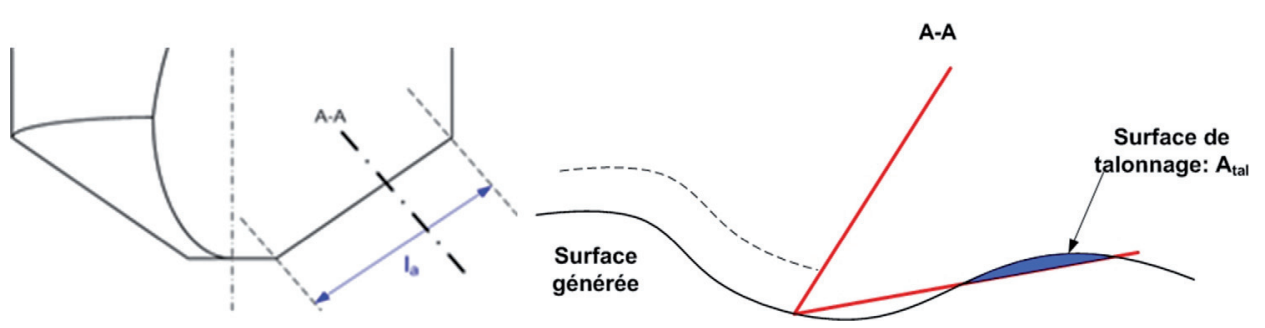

Fig. 7. Représentation du talonnage.

\subsubsection{Modèle d'effort de talonnage}

Le phénomène de talonnage est un problème majeur dans les opérations d'usinage. Il apparaît lorsque la face en dépouille de l'outil vient en contact avec la surface usinée, générant ainsi un effort supplémentaire $F_{\text {tal }}$. En perçage conventionnel, il est possible de calculer une vitesse d'avance maximale $V_{\mathrm{zmax}}$ en fonction de l'angle en dépouille $\alpha$ et de la vitesse de coupe $V$ permettant d'éliminer ce phénomène de talonnage (Éq. (8)).

$$
V_{\mathrm{z} \max }=V \cdot \tan (\alpha) .
$$

Du fait des vibrations, en perçage vibratoire, la vitesse axiale instantanée du foret peut être très importante. De plus la surface du fond du trou usiné n'est plus régulière mais se présente sous forme de vagues (Fig. 7). La combinaison de ces 2 facteurs fait que le phénomène de talonnage est très souvent présent et ce quel que soit l'affutage de l'outil (2 pentes, spiro-conique...) du fait de l'amplitude importante des vibrations devant l'avance de l'outil (l'amplitude des vibrations est comprise entre 2 et 3 fois l'avance de l'outil). Il peut donc plus être négligé. Wu [9] modélise l'effort dû au talonnage comme étant proportionnel au volume de matière déformée (Éq. (9)).

$$
F_{\text {tal }}=K_{\text {tal }} \cdot V_{\text {tal }}
$$

Le volume de matière déformée peut quant à lui s'exprimer comme étant le produit de la section de matière déformée $A_{\text {tal }}$ par la longueur de l'arête de coupe $l_{\text {a }}$ (coupe A-A de la Fig. 7). L'angle de dépouille $\alpha$ varie le long de l'arête de coupe et la section de matière déformée $A_{\text {tal }}$ n'est pas constante le long de l'arête. L'utilisation du découpage de la zone de coupe en segments $i$ va donc permettre d'estimer le talonnage de chaque subdivision de longueur $l_{\text {ai }}$ en évaluant l'aire de matière déformée par zone $A_{\text {tali }}$ (Éq. (10)). L'effort total de talonnage s'exprime à l'aide de l'équation (11).

$$
\begin{aligned}
\Delta F_{\text {tali }} & =K_{\text {tal }} \cdot A_{\text {tali }} \cdot I_{\text {ai }} \\
F_{\text {tal }} & =N_{\mathrm{c}} \sum_{i=1}^{n} \Delta F_{\text {tali }}
\end{aligned}
$$

\subsubsection{Effort de coupe dans la zone de coupe secondaire}

Le phénomène de coupe dans cette zone est aussi un phénomène d'enlèvement de matière comme dans la zone de coupe principale, mais avec des angles de coupe fortement négatifs. Il est donc possible d'utiliser le modèle proposé par Yang [7] avec un nouveau coefficient spécifique $K_{2 \text { t }}$ (Éq. (12)). Cette zone étant de très petite taille, environ 1/10 du rayon du foret, le coefficient $K_{2 \mathrm{t}}$ est considéré constant. Aucune segmentation de cette partie n'est nécessaire.

$$
F_{\mathrm{c}}^{2}=N_{\mathrm{c}} \cdot K_{2 \mathrm{t}} \cdot\left(h \cdot l_{2}\right)^{q}
$$

\subsubsection{Effort de coupe dans la zone centrale}

Dans cette zone, la vitesse de coupe est quasi nulle. Le phénomène d'extrusion apparaît. La matière est repoussée par la zone centrale du foret provoquant ainsi un effort important. Yang [7] propose un modèle de ce phénomène d'extrusion décrit par l'équation (13).

$$
F_{\mathrm{c}}^{3}=K_{3 \mathrm{t}} \cdot h \cdot R_{3}
$$

Le paramètre $R_{3}$ représente le rayon de la zone d'extrusion. Ce rayon n'est pas constant et dépend de l'avance instantanée de l'outil (cette avance instantanée est égale à l'épaisseur instantanée de copeau $h$ définie au début de l'article) et de l'angle de pointe du foret $\delta$ (Éq. (14)). $K_{3 \mathrm{t}}$ est un coefficient caractérisant la pression spécifique d'extrusion.

$$
R_{3}=\frac{h}{2 \cdot \tan \left(\frac{\pi-\delta}{2}\right)}
$$

\section{Simulation du perçage vibratoire}

La principale difficulté du perçage vibratoire réside dans le choix des paramètres de coupe permettant d'obtenir des vibrations suffisantes pour fragmenter le copeau. Pour cela, les modèles développés dans la partie 2 ont été introduits dans un simulateur développé sous Matlab ${ }^{\circledR}$. Le simulateur permet, pour une configuration donnée, de fournir des informations sur les vibrations du foret, l'épaisseur du copeau, les efforts de coupe dans les différentes zones et les efforts de talonnage.

Le principe de base du simulateur repose sur l'intégration numérique de l'équation différentielle (Éq. (1)) à l'aide d'un schéma d'intégration numérique de Newmark. Chaque tour de foret est discrétisé en $M$ incréments. À chaque incrément, un calcul de 


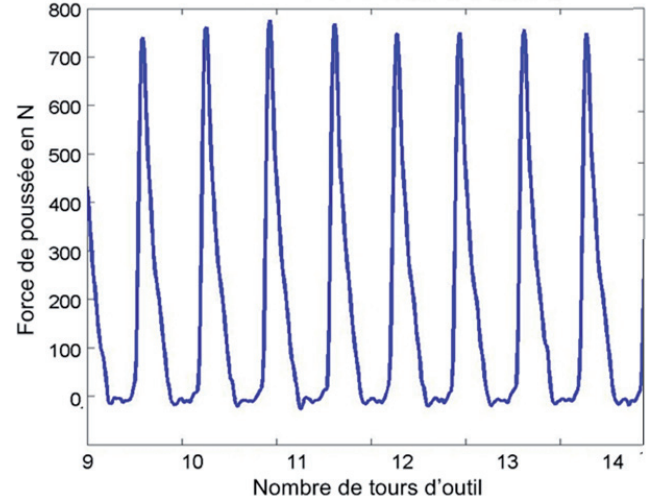

Fig. 8. Effort de poussée expérimental sur 5 tours de foret.

l'équilibre de l'équation différentielle donne la position de l'arête de coupe et la géométrie de la surface usinée est alors actualisée.

\subsection{Validation du simulateur}

Une campagne d'essai avec la tête de perçage vibratoire a permis de calibrer les différents paramètres du simulateur permettant ainsi une validation de celui ci. Prenons par exemple un perçage dans la configuration suivante : foret hélicoïdal GUHRING de diamètre de $4 \mathrm{~mm}$ revêtu TiN avec géométrie de pointe de type dépouille conique, une avance de $0,06 \mathrm{~mm} \cdot \mathrm{tr}^{-1}$, une vitesse de rotation de $5570 \mathrm{tr} \cdot \mathrm{min}^{-1}$ avec le porte-outil de perçage vibratoire ayant une masse mobile de $2,450 \mathrm{~kg}$ et une raideur de $259 \mathrm{~N} . \mathrm{mm}^{-1}$. La matière percée est de l'acier C38. Le poste de travail a été instrumenté avec un dynamomètre Kistler permettant de mesurer les efforts de poussée.

L'effort de poussée est présenté en figure 8. Les vibrations de l'outil sont bien régulières. L'effort de poussée mesuré s'annule de manière très régulière. Ce phénomène est normal puisque le foret vibre et l'arête de coupe saute hors de la matière (effort nul à ce moment) puis repénètre dans la matière. Les vibrations de l'outil sont très régulières avec une fréquence de 1,8 vibrations par tour de foret. L'amplitude des efforts est de 750 N. Le taux de coupe représentant le rapport entre le temps effectif d'enlèvement de matière et le temps total d'usinage est alors calculé. Ce taux de coupe mesuré est de $40 \%$. Un taux de coupe faible a pour conséquence une bonne fragmentation du copeau qui a une forme bien spécifique au perçage vibratoire (Fig. 10).

Les résultats de simulation, dans les mêmes conditions, montrent que l'amplitude des efforts est sensiblement identique : $750 \mathrm{~N}$ d'amplitude pour l'essai et $700 \mathrm{~N}$ d'amplitude pour la simulation (Fig. 9). Le comportement vibratoire de l'outil est identique avec 1,8 oscillations par tour et le taux de coupe vaut $36 \%$. D'autres essais ont été effectués avec des conditions de coupe différentes. Les résultats obtenus montrent une bonne corrélation avec les résultats de simulation. Le simulateur peut donc être

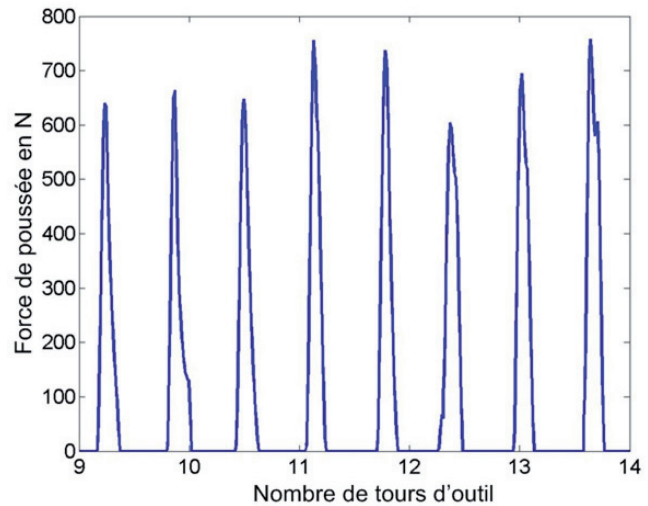

Fig. 9. Effort de poussée simulé sur 5 tours de foret.

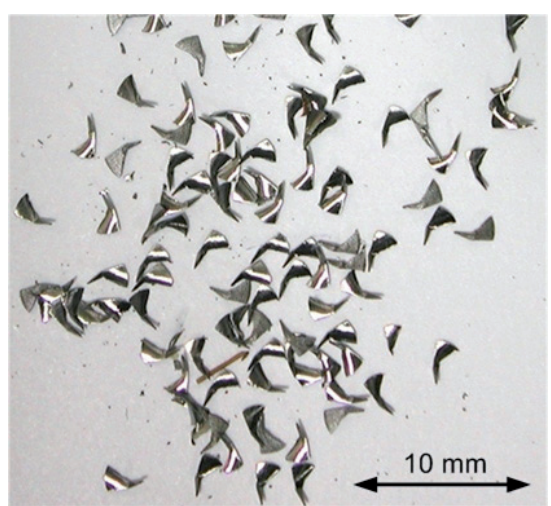

Fig. 10. Copeaux générés en perçage vibratoire.

utilisé pour prédire l'influence des différents paramètres sur le comportement du porte-outil de perçage vibratoire.

\section{Influence du talonnage sur les vibrations de l'outil}

Le simulateur peut maintenant être utilisé pour étudier l'influence du talonnage sur le mouvement vibratoire de l'outil. Deux simulations ont été effectuées : une avec un foret ayant un angle de dépouille de 8 degrés (Fig. 11) et l'autre avec un angle de dépouille de 3 degrés dans le but d'augmenter le phénomène de talonnage (Fig. 12). L'arête principale (zone 1) a été discrétisée en 3 parties appelées respectivement zone 1a, zone $1 \mathrm{~b}$ et zone 1c. Tous les autres paramètres restent inchangés par rapport à la configuration prise lors de la validation du simulateur.

Avec un angle de dépouille de $8^{\circ}$ (Fig. 11a), l'amplitude des vibrations créées par la coupe est de $400 \mu \mathrm{m}$. Avec un angle de dépouille de $3^{\circ}$ (Fig. 12a), l'amplitude des vibrations dans la zone stabilisée est réduite à $50 \mu \mathrm{m}$. Cette nette diminution de l'amplitude montre que l'amortissement des vibrations dû au talonnage n'est pas négligeable. Cet amortissement va se répercuter sur l'épaisseur du copeau (175 $\mu$ m Fig. 11b et $80 \mu \mathrm{m}$ Fig. 12b) et sur le taux de coupe (passant de $40 \%$ à $71 \%$ ). 


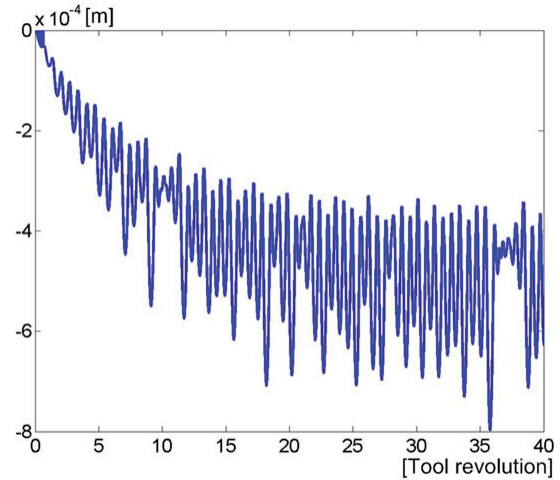

a. Amplitude des vibrations

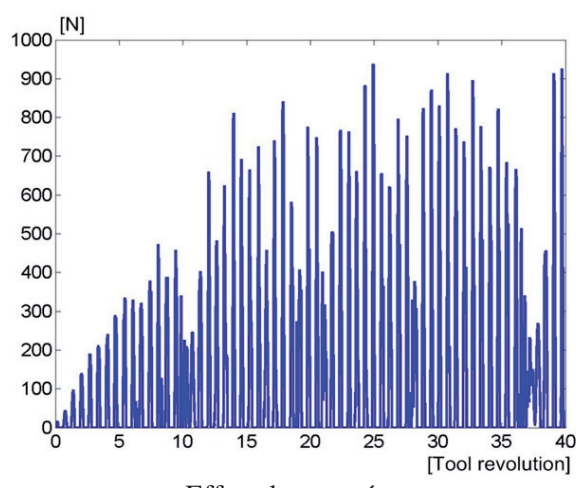

c. Effort de poussée

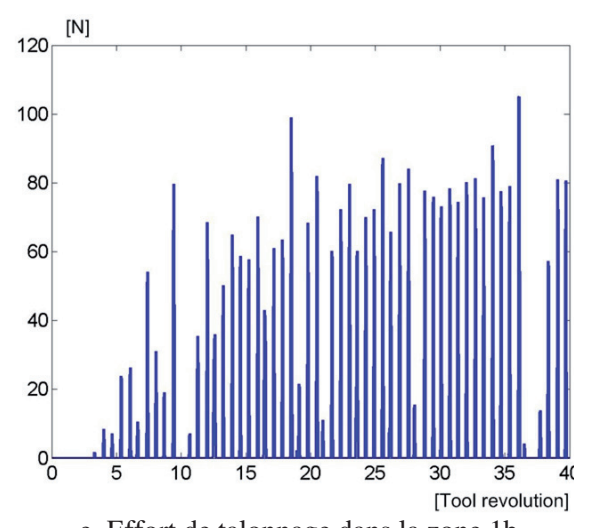

e. Effort de talonnage dans la zone $1 \mathrm{~b}$

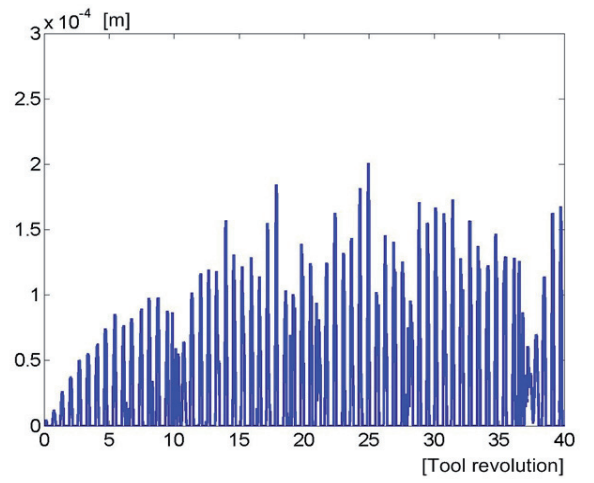

b. Epaisseur du copeau

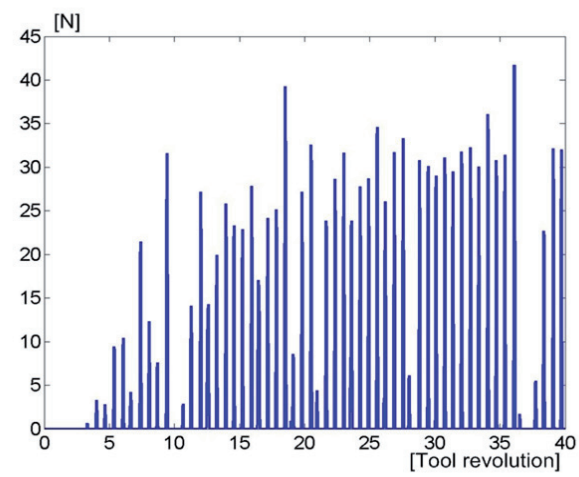

d. Effort de talonnage dans la zone 1a

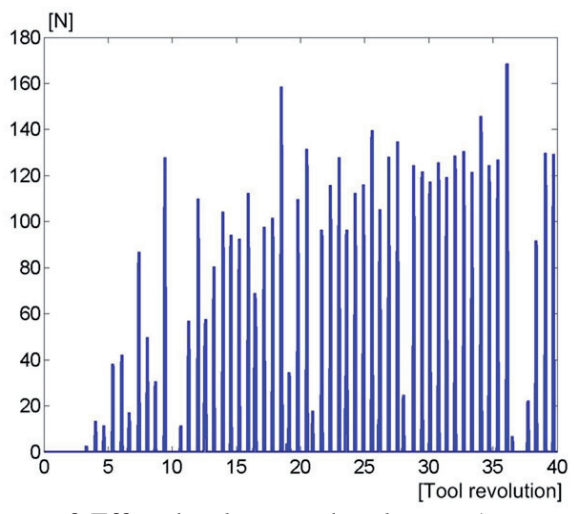

f. Effort de talonnage dans la zone 1c

Fig. 11. Simulation de perçage avec un outil ayant un angle de dépouille de 8 degrés.

Les efforts dus au talonnage augmentent aussi de manière significative. Avec un angle de dépouille de 8 degrés, les efforts générés par le talonnage sont de $35 \mathrm{~N}$ dans la zone 1a, de $80 \mathrm{~N}$ dans la zone $1 \mathrm{~b}$ et de $120 \mathrm{~N}$ dans la zone 1c (Figs. 11d-f). Avec un angle en dépouille de $3^{\circ}$, les efforts de talonnage sont bien plus importants : $60 \mathrm{~N}$ dans la zone 1a, $160 \mathrm{~N}$ dans la zone $1 \mathrm{~b}$ et $250 \mathrm{~N}$ dans la zone 1c (Figs. 12d-f).

Ces simulations montrent que le talonnage va engendrer des efforts supplémentaires importants qui vont avoir pour effet d'amortir les vibrations. L'amortissement des vibrations va se traduire par une augmentation du taux de coupe. Le copeau est alors moins bien fractionné et son évacuation devient délicate. Les effets du perçage vibratoire sont donc éliminés. Il est donc important de trouver des conditions de coupe permettant d'obtenir des vibrations suffisantes pour fragmenter le copeau tout en minimisant le phénomène de talonnage. Le simulateur numérique va permettre de trouver le point de fonctionnement optimal pour une opération de perçage (type de foret, diamètre, matériau), limitant ainsi le temps de mise au point.

\section{Conclusion}

L'utilisation du perçage vibratoire pour des perçages de petits diamètres améliore considérablement la productivité principalement car la bonne fragmentation du 

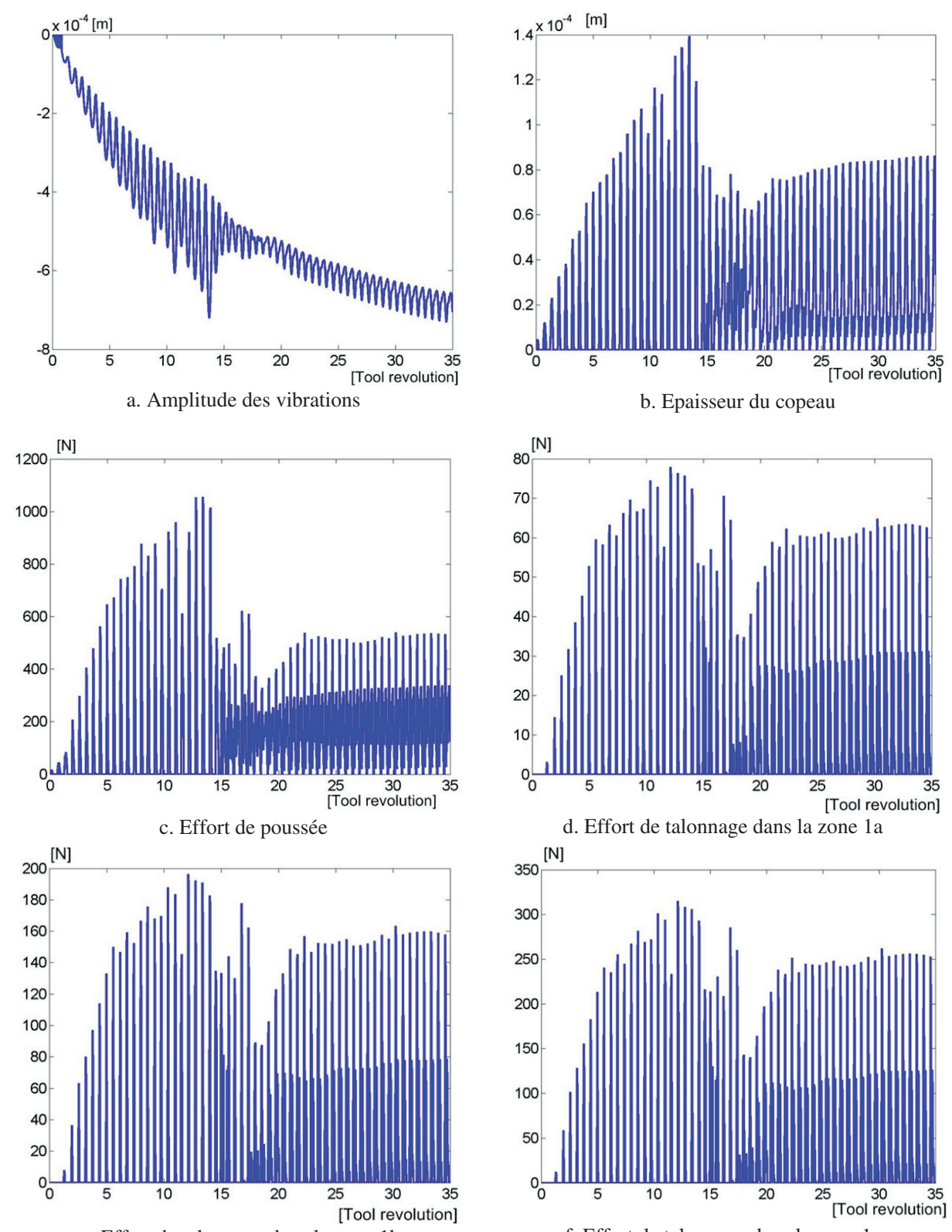

d. Effort de talonnage dans la zone 1a

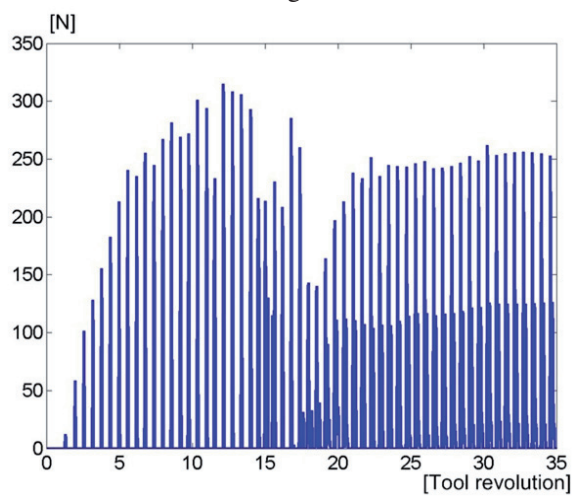

f. Effort de talonnage dans la zone 1c

Fig. 12. Simulation avec un outil ayant un angle de dépouille de 3 degrés.

copeau entraîne une élimination des cycles de débourrage. Cette technique nécessite un choix des paramètres de coupe permettant de générer, via le ressort spécifique placé dans le porte-outil, des vibrations suffisantes pour fragmenter le copeau tout en conservant une durée de vie acceptable pour l'outil. L'application de cette technique au niveau industriel présente donc un réel intérêt par un gain de productivité lié à l'élimination des cycles de débourrage.

Pour aider au choix des paramètres de coupe, un simulateur intégrant les différents modèles choisis a été développé. Ce simulateur permet d'établir pour chaque configuration l'existence de vibrations mais aussi de donner des informations sur les efforts de coupe, l'épaisseur du copeau et l'amplitude de vibrations.
Le perçage vibratoire est très sensible au phénomène de talonnage. En effet, du fait des vitesses instantanées élevées et de la forme de la surface usinée, le talonnage apparaît fréquemment. Ce talonnage agit comme un amortisseur supplémentaire et va absorber les vibrations. L'amplitude des vibrations n'est plus suffisante, le copeau ne se fragmente donc plus correctement et une absence de fragmentation du copeau se traduit très souvent par le bris de l'outil. Le simulateur permet donc de choisir des conditions de coupe garantissant une bonne fragmentation du copeau.

Remerciements. Remerciements au CETIM qui finance la partie expérimentale de ce travail de thèse par le biais d'un contrat entre CETIM, le LTDS (Enix) et le laboratoire G-SCOP. 


\section{Références}

[1] A. Gouskov, S.A. Voronov, S.A. Batzer, Chatter synchronization in Vibratory Drilling, ASME 108 (2000) 263-270

[2] C. Suciu, Analytical investigation of an active-control squeeze film damper, Rotrib'96, Editura Tehnicã, Bucharest 18 (1996) 16-26

[3] P.N. Chabra, B. Ackroyd, W.D. Compton, Low Frequency Modulation-assisted Drilling Using Linear Drives, J. Eng. Man. 216 (2002) 321-330

[4] S. Tichkiewitch, G. Moraru, D. Brun-Picard, A. Gouskov, Self-Excited Vibration Drilling Models and Experiments, Annals of the CIRP 51 (2002) 311-314

[5] G. Peigne, E. Kamnev, D. Brissaud, A. Gouskov, Selfexcited vibratory drilling: a dimensionless parameter approach for guiding experiments, J. Eng. Man. 219 (2005) 73-85

[6] H. Paris, S. Tichkiewitch, G. Peigne, Modelling the vibratory drilling to foresee cutting parameters, Annals of the CIRP 54 (2004) 367-370

[7] J.A. Yang, J. Venkatraman, D. Ruxu, A new dynamic model for drilling and reaming process, J. Mach. Tools Man. 42 (2001) 299-311

[8] D.A. Stephenson, P. Bandyopadhyay, Processindependent force caracterization for metal-cutting simulation, Transactions of the ASME, J. Eng. Ind. 119 (1997) 86-94

[9] D.W. Wu, A new approach of formulating the transfer function for dynamic cutting process, ASME J. Eng. Ind. 111 (1989) 37-47 